\title{
Prognostic Value of Bone Formation and Resorption Proteins in Heterotopic Ossification in Critically-Ill Patients. A Single-Centre Study
}

\author{
Alice Georgia Vassiliou ${ }^{1}$, Edison Jahaj ${ }^{1}$, Zafeiria Mastora ${ }^{1}$, Ioannis Karnezis², loanna \\ Dimopoulou ${ }^{1}$, Stylianos E. Orfanos ${ }^{1}$, Anastasia Kotanidou ${ }^{*}$ \\ 1 First Department of Critical Care Medicine and Pulmonary Services, School of Medicine, National and Kapodistrian \\ University of Athens, Evangelismos Hospital, Athens, Greece \\ 2 Department of Orthopedics, Evangelismos Hospital, Athens, Greece
}

\begin{abstract}
Introduction: A potential complication in critically ill patients is the formation of bone in soft tissues, termed heterotopic ossification. The exact pathogenetic mechanisms are still undetermined. Bone morphogenetic proteins induce bone formation, while signalling through the receptor activator of nuclear factor kappa-B (RANK) and its ligand (RANKL), regulates osteoclast formation, activation, and survival in normal bone modelling and remodelling. Osteoprotegerin protects bone from excessive bone loss by blocking RANKL from binding to RANK. Aim: The study aimed to investigate these molecules as potential prognostic biomarkers of heterotopic ossification development in critically ill patients. Materials and Methods: In this prospective observational study, BMP-2, RANKL, and osteoprotegerin were measured by ELISA in twenty-eight critically-ill, initially non-septic patients, on admission to an ICU, seven days postadmission, and thirty days after ICU discharge. Results: In the critically-ill cohort, nine of the twenty-eight patients developed heterotopic ossification up to the 30-day follow-up time-point. The patients who developed heterotopic ossification exhibited significantly reduced BMP-2 and RANKL levels on ICU admission, compared to patients who did not; Osteoprotegerin readings were similar in both groups. Conclusions: Critically-ill patients who will subsequently develop heterotopic ossification, have significantly lower BMP-2 and RANKL levels at the time of ICU admission, suggesting that these proteins may be useful as prognostic markers for this debilitating condition.
\end{abstract}

Keywords: bone morphogenetic proteins, critically-ill, heterotropic ossification, osteoprotegerin, receptor activator of nuclear factor kappa-B ligand

Received: 26 August 2020 / Accepted: 26 November 2020

\section{INTRODUCTION}

Heterotopic ossification is defined as the development of mature lamellar bone in soft tissues [1]. It is a condition affecting a substantial minority of critically-ill patients even after discharge from hospital. The cause of heterotopic ossification has not yet been entirely determined. Bone morphogenetic protein (BMP) signalling is thought to play a central part in the heterotopic ossification process. BMPs are members of the transforming growth factor- $\beta$ (TGF- $\beta$ ) family that regulate proliferation, differentiation, apoptosis, and motility of various cell types in the course of development and tissue homeostasis, including regulation of bone homeostasis $[2,3]$.
BMP-2, BMP-4, BMP-6, BMP-7, and BMP-9 have been shown to induce adipogenic and osteogenic differentiation in vitro and in vivo $[4,5]$ while a central role for BMP signalling has been demonstrated in trauma-induced heterotopic ossification [6]. Likewise, BMP-2 has been demonstrated to cause heterotopic ossification in a murine model studying spinal cord injury (SCI) [7]. Thus, it has been suggested that the BMP signalling pathway may be used as a target in the treatment of bone diseases [2]. On the other hand, signalling through the receptor activator of nuclear factor kappa-B (RANK) and its ligand (RANKL) regulates osteoclast formation, activation, and survival in normal bone modelling and remodelling $[8,9]$. Osteoprote- 
gerin prevents RANKL from binding to RANK, thus preventing excessive bone loss [10].

In critically-ill patients, the timely diagnosis of heterotopic ossification has been a challenge. Age, sex, and previous trauma and surgery have been proposed as predisposing factors. Laboratory data, such as increased serum alkaline phosphatase are considered non-specific, while elevated serum creatine kinase has been suggested to be associated with a more aggressive course of heterotopic ossification [11]. Until now, there is no way to identify critically-ill patients who will develop this condition.

The present study aimed to investigate serum biomarkers as possible indicators of heterotopic ossification development in a cohort of ICU patients.

\section{MATERIALS AND METHODS}

The study was approved by the Hospital's Ethics Committee (study approval number 80 - 1/2/2010). All procedures conformed with the Helsinki Declaration. Informed written consent was obtained from all patients' next-of-kin before any study procedure.

\section{Study Design}

A prospective, observational study of critically ill patients treated in one academic, multidisciplinary ICU.

\section{Study Population}

All consecutive admissions to the intensive care unit of the "Evangelismos" General Hospital, Athens, Greece, were screened for eligibility over fifteen months from the 16th of June 2012 to the 27th of September 2014.

Exclusion criteria were:

- sepsis within the first 24 hours of ICU admission [12]

- BMI greater than $35 \mathrm{~kg} / \mathrm{m}^{2}$

- age less than 18 years

- pregnancy

- total ICU stay less than three days

- end-stage cancer or brain death

- no need for mechanical ventilation

- re-admission or transfer from another ICU

- contagious diseases (human immunodeficiency virus, hepatitis)

- oral intake of corticosteroids at an equivalent dosage of $\geq 1 \mathrm{mg} / \mathrm{kg}$ prednisone per day for more than one month
Out of sixty-four patients screened for eligibility, thirty-six patients were initially included in the study based on the above criteria. The study number was also reduced by patients refusing consent. Finally, twentyeight critically-ill patients, twenty-one male and seven female, completed the study, as an additional eight patients were lost to follow-up (Figure 1).

The patients enrolled in the study were mainly neurosurgical and trauma patients. Clinical and laboratory data were recorded for all enrolled patients.

ICU admission anthropometric data, severity scores and medical history were noted.

Blood samples were drawn at three-time points:

- on ICU admission (baseline)

- Seven days following admission

- Thirty days after ICU discharge, approximately two months post-admission to the ICU.

The demographics and clinical characteristics of the patients are listed in Table 1.

Patients were followed-up for 30 days following discharge from the ICU.

Patients were allocated to two groups based on the development of heterotopic ossification at any point during their ICU stay or during the 30-day follow-up period, as verified by $\mathrm{X}$-rays.

This resulted in two groups:

- Group 1. heterotopic ossification -positive, $\mathrm{N}=9$

- Group 2. heterotopic ossification -negative, $\mathrm{N}=19$

None of the patients had heterotopic ossification on admission.

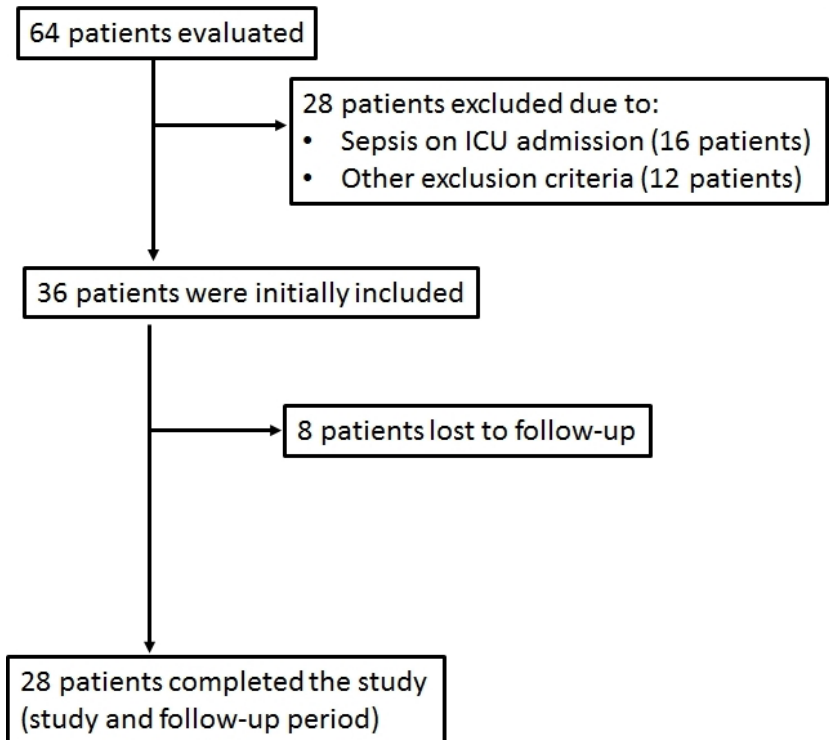

Fig. 1. Study enrolment flow chart 
Table 1.Demographics on ICU admission and important outcomes of the patients who developed heterotopic ossification (HO) (HO-positive) versus those who did not (HO-negative).

\begin{tabular}{|c|c|c|c|}
\hline Demographics/Outcomes & HO-positive patients & HO-negative patients & p-value \\
\hline Number of patients ( $\mathrm{N}$ ) & 9 & 19 & \\
\hline Age & $51(19)$ & $51(14)$ & ns \\
\hline Sex & & & ns \\
\hline Male & 7 & 14 & \\
\hline Female & 2 & 5 & \\
\hline APACHE II, mean (SD) & $16(6)$ & $17(5)$ & ns \\
\hline SOFA, mean (SD) & $7(1)$ & $7(2)$ & ns \\
\hline $\operatorname{CRP}(\mathrm{mg} / \mathrm{dl})$, (median, IQR) & $5.1(1.6-12.0)$ & $5.5(1.1-8.8)$ & ns \\
\hline РСТ (ng/ml), (median, IQR) & $1.1(0.2-2.5)$ & $0.1(0.1-0.8)$ & ns \\
\hline Creatinine $(\mathrm{mg} / \mathrm{dl})$, mean (SD) & $0.7(0.2)$ & $0.9(0.3)$ & ns \\
\hline Vitamin D (ng/ml), (median, IQR) & $6.8(4.2-13.6)$ & $12.7(6.5-16.8)$ & ns \\
\hline Diagnosis & & & ns \\
\hline Medical & $0 \%$ & $15.8 \%$ & \\
\hline Surgery/Trauma & $100 \%$ & $84.2 \%$ & \\
\hline Medical & 0 & 3 & \\
\hline Cerebral abscess & - & 1 & \\
\hline Subarachnoid haemorrhage & - & 1 & \\
\hline Cerebral haemorrhage & - & 1 & \\
\hline Surgical Emergency & 5 & 8 & \\
\hline Thoracic & 0 & 0 & \\
\hline Neurosurgery & 5 & 8 & \\
\hline Abdominal & 0 & 0 & \\
\hline Trauma & 4 & 8 & \\
\hline Comorbidities & 3 & 7 & ns \\
\hline CAD & 1 & & \\
\hline Arterial Hypertension & 1 & 6 & \\
\hline Hypothyroidism & 1 & 2 & \\
\hline Mechanical ventilation (days) & $20(17-25)$ & $22(16-30)$ & ns \\
\hline ICU Length of Stay (days) & $29(21-35)$ & $30(22-40)$ & ns \\
\hline Sepsis & $66.7 \%$ & $84.2 \%$ & ns \\
\hline Day of sepsis & $10(7-12)$ & $8(5-10)$ & ns \\
\hline
\end{tabular}

\section{Measurement of Biomarkers}

BMP-2, 4, 6, 7, RANKL, and OPG were measured in plasma samples by enzyme-linked immunosorbent assay (ELISA), according to the manufacturers' instructions. BMP-2, 4, 7, RANKL, and Osteoprotegerin was purchased from Elabscience [Elabscience, Houston, USA] and BMP-6 from Sigma-Aldrich [Sigma-Aldrich, Saint Louis, USA].

On Day 7 post-admission to the ICU, and Day 30 of the follow-up period, X-rays of the large joints, knee, elbow, shoulder, pelvis, and hip, were obtained from all patients to verify the presence of heterotopic ossification.
Measurements were performed using the Brooker classification for the hip and the Graham classification for the elbow, by a specialist orthopedist who was blinded to the biochemical marker data.

\section{Statistical Analysis}

Individual values, mean (SD) for normally distributed variables, or median with interquartile range (IQR) for variables with skewed distribution, are given.

The Student's t-test, the non-parametric MannWhitney test, one-way ANOVA for repeated measures or Kruskal-Wallis ANOVA followed by Dunn's post hoc test were used, accordingly. 
The chi-square test or Fisher's exact were used to examine associations between qualitative variables. Receiver operating characteristic (ROC) curves were plotted after that using $\mathrm{HO}$ development as the classification variable and BMP-2 and RANKL levels on ICU admission as prognostic variables. The optimal cut-off value for predicting heterotopic ossification was calculated as the point with the greatest sensitivity and specificity.

The power of the study was calculated post-hoc.

GraphPad Prism 6 (GraphPad Software, Inc) was used for the analyses.

The significance level was set at $\alpha=0.05$.

\section{RESULTS}

Among the 28 critically-ill adult patients who completed the study, $75 \%$ were male, and $25 \%$ were female. The mean (SD) age of the population was 51 (16) years old.

The mean admission acute physiology and chronic health evaluation (APACHE) II score was 17 (5) and of the sequential organ failure assessment (SOFA) score 7 (2). The overall ICU mortality rate amongst our study patients was 7\%. Approximately $90 \%$ of the patients were trauma patients (43\%) and surgical (46\%) patients. The remainder had diagnosed medical conditions.

About $80 \%$ of the patients developed sepsis during their ICU stay. Demographics, severity scores, and outcomes of the two patient groups are listed in Table 1.

$\mathrm{X}$-rays showed that during the 30 -day follow-up period, nine patients developed heterotopic ossification. Seven patients developed mild bilateral heterotopic ossification (Brooker grade 1 or 2), while two developed mild unilateral heterotopic ossification (Brooker grade 1). Four of these patients also developed Graham Class I heterotopic ossification in one elbow.

Human BMP-2, 4, 6, 7, RANKL, and OPG were measured simultaneously by enzyme-linked immunoassay (ELISA) in the three time-point samples of all patients.

Nine of the twenty-eight patients developed heterotopic ossification during the follow-up period. The results revealed that the patients who developed heterotopic ossification had significantly reduced ICU admission BMP-2 levels compared to patients who did not [566.7 (216.7-883.3) pg/ml vs 1300 (566.7-2817) $\mathrm{pg} / \mathrm{ml}$, respectively; $\mathrm{p}=0.037$ ] (Figure 2a).
ICU admission RANKL levels were also lower in the patients who subsequently developed heterotopic ossification [1.495 (0.285-2.465) ng/ml vs. 2.465 (1.535$6.67) \mathrm{ng} / \mathrm{ml} ; \mathrm{p}=0.048]$ (Figure $2 \mathrm{~b}$ ).

The OPG admission levels and the RANKL: OPG ratio were similar in both groups (Figure $2 \mathrm{c}$ and $\mathrm{d}$ ).

In the patients who developed heterotopic ossification, the levels of BMP-2 were increased by Day 7, whereas by Day 30 of the follow-up period, they tended to increase $(p=0.064)$ (Figure 3a).

The patients who did not develop heterotopic ossification had stably elevated BMP-2 levels during the entire study period (Figure 4a).

A small rise was observed in RANKL levels in the heterotopic ossification patients on Day 30 of the follow-up period (Figure 3b). OPG levels and the RANKL: OPG ratio remained unaltered during the entire study period in both groups (Figure $3 \mathrm{c}$ and $\mathrm{d}$ and Figure $4 \mathrm{c}$ and $d$ ).

BMP-4, 6, and 7 were only detectable in a small per cent of the patients. This precluded any further statistical analysis.

A ROC curve was generated to determine the prognostic accuracy of BMP-2 and RANKL admission levels.

The area under the ROC curve (AUC) and 95\% confidence intervals (CI) for detecting the main outcome, i.e. heterotopic ossification development, was estimated for BMP-2 at $0.823(0.623-1.023, \mathrm{p}=0.038)$ and for RANKL at 0.767 (0.557-0.977, p= 0.048) (Figure 5). BMP-2 at $833 \mathrm{pg} / \mathrm{ml}$ showed a sensitivity of $80 \%$ and a specificity of $69.2 \%$, while RANKL at $1.52 \mathrm{ng} / \mathrm{ml}$ showed a sensitivity of $71.4 \%$ and a specificity of $80 \%$.

Additional parameters analysed in the cohort with regards to their association with heterotopic ossification development, included age, sex, diagnostic category, APACHE II, SOFA score, procalcitonin (PCT), C-reactive protein (CRP), creatinine, and vitamin D levels, all recorded on ICU admission (Table 1).

There was no significant difference between the two groups concerning the quantitative variables (Student's $\mathrm{t}$-test or non-parametric Mann-Whitney test $(\mathrm{p}>0.05)$ or the qualitative variables (chi-square test or the Fisher's exact ( $\mathrm{p}>0.05)$ (Table 1$)$.

\section{DISCUSSION}

In the present study, we demonstrated that in a critically-ill cohort, patients who will develop heterotopic 


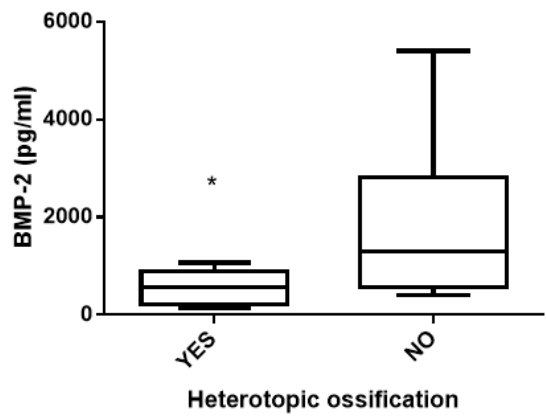

c

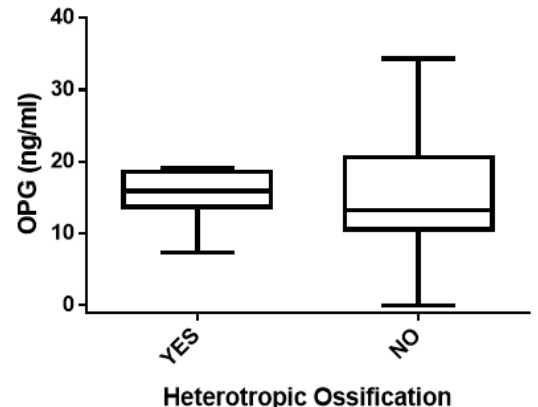

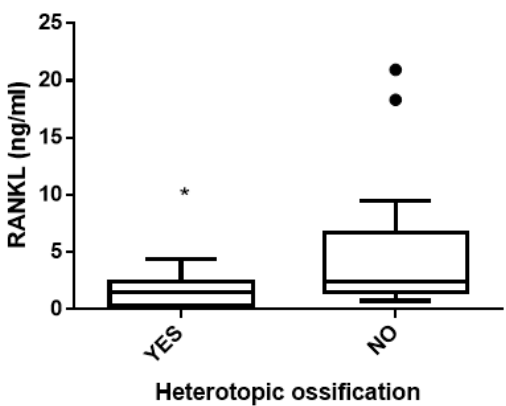

d

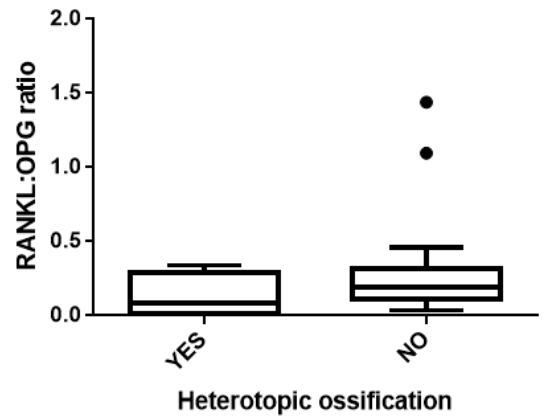

Fig. 2. BMP-2, RANKL and Osteoprotegerin levels on admission. a) BMP-2, b) RANKL, c) Osteoprotegerin and d) RANKL: Osteoprotegerin ratio were measured in 28 critically ill, initially non-septic patients on ICU admission. Patients were subsequently categorised as heterotopic ossification-positive (those who subsequently developed heterotopic ossification, yes, $\mathrm{N}=9$ ) and heterotopic ossification -negative (those who did not develop heterotopic ossification, no, $\mathrm{N}=19$ ). Two-group comparisons were performed with the non-parametric Mann-Whitney test, $* p<0.05$. Data are presented as box plots. Line in the box, median value; box edges, 25th to 75th centiles; whiskers, range of values; bullet points, outliers. BMP-2= bone-morphogenetic protein 2, RANKL= receptor activator of nuclear factor kappa-B ligand, OPG= osteoprotegerin.

a

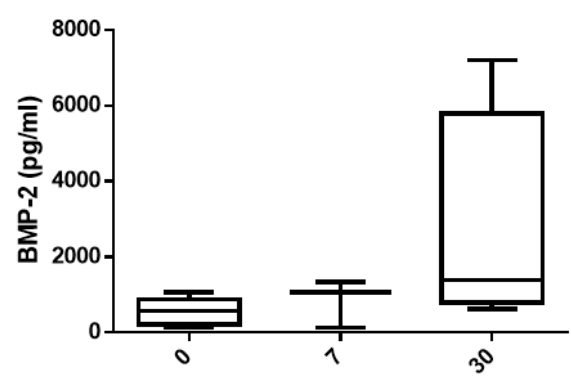

C

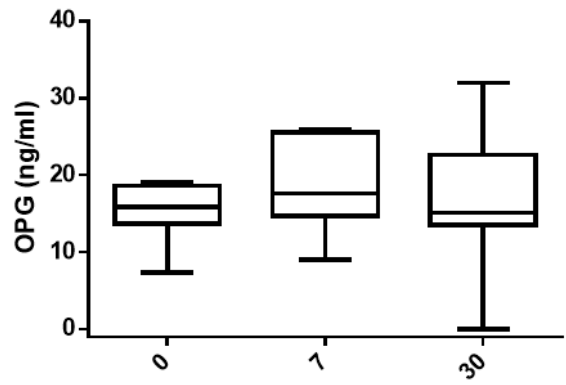

b

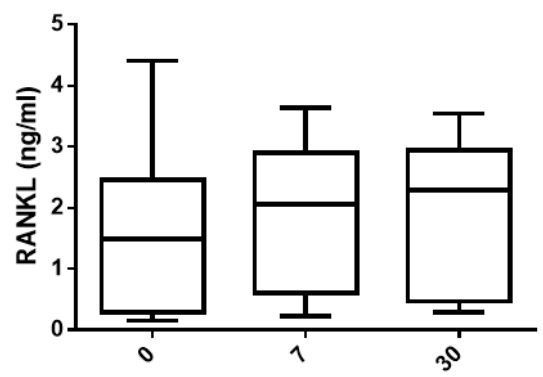

d

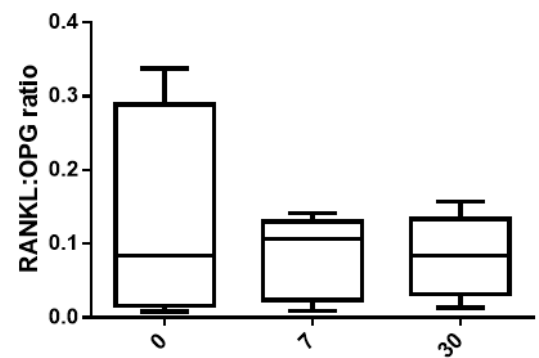

Fig.3. Time course of BMP-2, RANKL and OPG levels during ICU stay and the follow-up period in the patients who developed heterotopic ossification. a) BMP-2, b) RANKL, c) OPG and d) RANKL: OPG ratio were measured in 9 patients who developed heterotopic ossification on ICU admission (0), and thereafter seven days post-admission (7) and 30 days following ICU discharge (30). Data are presented with box plots. Line in the box, median value; box edges, 25th to 75th centiles; whiskers, range of values. Comparisons were performed using one-way ANOVA followed by Dunn's multiple comparisons. BMP-2= bone-morphogenetic protein 2, RANKL= receptor activator of nuclear factor kappa-B ligand, OPG = osteoprotegerin. 

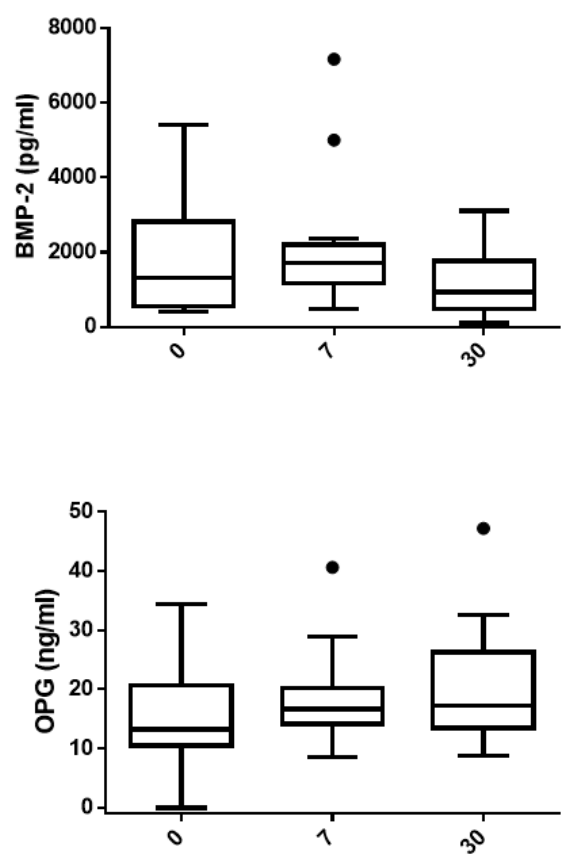

b

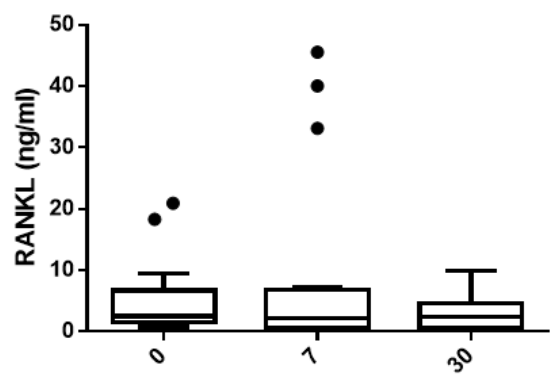

d

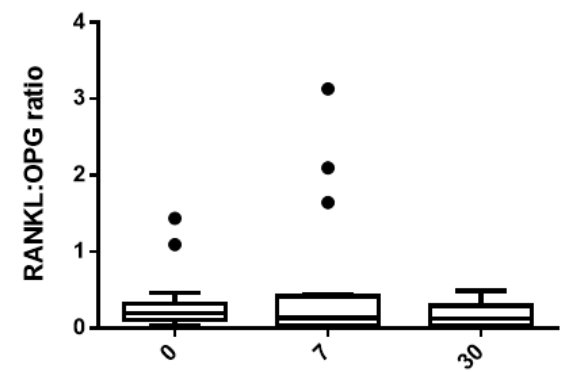

Fig. 4. Time course of BMP-2, RANKL and OPG levels during ICU stay and the follow-up period in the patients who did not develop heterotopic ossification. a) BMP-2, b) RANKL, c) OPG and d) RANKL: OPG ratio were measured in 19 patients who did not develop heterotopic ossification on ICU admission (0), and thereafter seven days post-admission (7) and 30 days following ICU discharge (30). Data are presented with box plots. Line in the box, median value; box edges, 25th to 75th centiles; whiskers, range of values; bullet points, outliers. Comparisons were performed using one-way ANOVA followed by Dunn's multiple comparisons. BMP-2= bone-morphogenetic protein 2, RANKL= receptor activator of nuclear factor kappa-B ligand, OPG= osteoprotegerin.

a

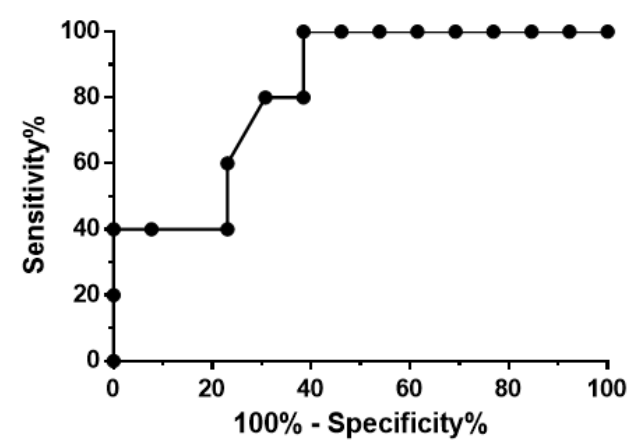

b

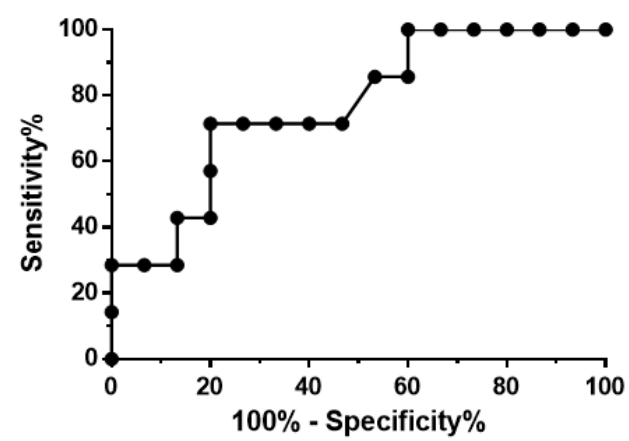

Fig. 5. Receiver operating characteristic (ROC) curve analysis. ROC curves were generated to determine the prognostic accuracy of a) BMP-2 and b) RANKL; the corresponding areas under the curve (AUC) and 95\% confidence intervals (CI) were estimated as follows: BMP-2 at $0.823(0.623-1.023, p=0.038)$; RANKL at $0.767(0.557-0.977, p=0.048)$. The levels of both molecules were estimated on ICU admission. BMP-2= bone-morphogenetic protein 2, RANKL= receptor activator of nuclear factor kappa-B ligand.

ossification have lower BMP-2 and RANKL levels on ICU admission compared to patients who do not develop heterotopic ossification. This indicates that these proteins might be useful as prognostic markers for this debilitating condition.

The results support the notion that a small but not negligible minority of ICU patients will develop heterotopic ossification during ICU stay or after discharge; no decisive way to identify these patients exists at present.

Furthermore, clinical diagnosis of heterotopic ossification is frequently impeded due to sedation and immobilisation of the patients. The X-ray confirmation of heterotopic ossification may be only evident a few weeks after the initial diagnosis is clinically speculated [13]. Thus, early detection of critically-ill patients who 
will develop heterotopic ossification constitutes a clinical challenge. In the present study, molecules involved in bone modelling and remodelling as potential indicators of heterotopic ossification development in ICU patients were investigated. The results indicated that low levels of BMP-2 and RANKL on ICU admission might serve as serum prognostic markers of heterotopic ossification in critically ill patients.

Most studies have focused on diagnostic indicators and treatments, and more specifically on the role of BMP-2 in inducing bone formation. Injection or the surgical implantation of BMPs and progenitor cells, expressing mainly BMP-2, can be used to induce heterotopic ossification formation [14-16].

The BMP-2 gene is expressed in the spinal ligaments of patients with ossification of the spinal ligament (OSL), and exogenous BMP-2 seems to stimulate osteogenic differentiation of spinal ligament cells [17]. It has also been proposed that BMP-2 may be implicated in the pathogenesis of osteoarthritic alterations or the repair process of temporomandibular joint internal derangement [18]. In foetal limb cultures, BMP-2 promoted chondrocyte hypertrophy and endochondral ossification [19], while two novel BMP-2 variants were identified in patients with thoracic ossification of the ligamentum flavum and functional assays revealed increased BMP-2 expression, elevated osteogenic marker expression, and augmented osteogenic differentiation [20]. Disrupting the BMP-signalling pathway has been proposed as a treatment strategy. Inhibition of BMP type I receptor (BMPR1) activity has been suggested in treating fibrodysplasia ossificans progressiva (FOP) and other heterotopic ossification syndromes associated with excessive BMP signalling [21], as well as blocking TGF- $\beta$ signalling to decrease the osteogenic transdifferentiation of FOP fibroblasts [22]. Others have demonstrated that the targeting of multiple BMP receptors averts trauma-induced heterotopic ossification [23]. The discovery of an mTOR-BMP signalling pathway has also been proposed as a possible molecular mechanism of heterotopic ossification that may constitute a novel therapeutic target for heterotopic ossification [24].

RANK, RANKL, and OPG recently appear as central regulators of bone metabolism [8]. Studies focusing on this pathway have shown the expression of RANKL and OPG in heterotopic vascular ossification [25], while OPG-deficient mice exhibit early-onset osteoporosis and arterial calcification [26].
The only study measuring factors belonging to these two pathways before developing heterotopic ossification demonstrated transient increases in osteoblast and osteoclast activity after total hip arthroplasty [27]. In this study development of heterotopic ossification was associated with an increase in the osteoblast markers $\mathrm{N}$-terminal pro-peptide of type-I procollagen (PINP), bone alkaline phosphatase (ALP) and osteocalcin (OC). However, the results showed that the osteoclast marker C-telopeptide of type-I collagen (CTX-I) was increased during the early phase of heterotopic ossification. Since this rise preceded heterotopic ossification development, the authors proposed that the pathogenesis of heterotopic ossification formation also involves early changes in osteoclast activity, rather than merely inappropriate new bone formation.

Normal bone remodelling involves osteoclasts removing the trenches and tunnels from the trabecular and cortical bones, respectively [10]. Subsequently, osteoblasts fill in these tunnels with new bone matrix. During normal bone remodelling, bone formation equals resorption [10]. In our critically-ill patients who will develop heterotopic ossification during their ICU stay or during the 30-day follow-up period, both osteoclast and osteoblast activity is reduced on ICU admission.

More specifically, our results showed that in the nine neurosurgical and trauma patients who were admitted to the ICU and developed heterotopic ossification, levels of BMP-2 were low, indicating that BMP-2 is probably not accumulated, and hence no new bone formation seems to occur. Furthermore, osteoclasts are not activated, as indicated by the low RANKL levels, hence old bone is not resorbed. In these patients, it becomes apparent that normal bone remodelling does not occur. Once heterotopic ossification is established, as verified by X-rays, both BMP-2 and RANKL return to levels comparable to the patients who did not develop heterotopic ossification.

The power of the study was calculated post-hoc, and the observed power was $60 \%$. The clinical assessment of heterotopic ossification was performed with X-rays, and not using MRI, which is considered a more sensitive method $[13,28]$. Also, variation between patients may limit the use of markers as individual prognostic factors, since bone turnover marker levels change at different time points in men and women and in metabolic bone disease $[29,30]$. Moreover, previous treatment with bisphosphonates, which may alter or inhibit 
osteogenesis in a chronic way, were not recorded, nor were the levels of the biomarkers before ICU admission.

\section{CONCLUSION}

In a critically-ill cohort, lower levels of both bone formation and resorption markers were observed on ICU admission in patients who eventually developed heterotopic ossification. The identification of reliable biomarkers might provide further insight into the pathogenesis of heterotopic ossification and may serve as an early prognostic tool for heterotopic ossification, especially given the absence of effective treatments.

\section{ACKNOWLEDGEMENTS}

The authors would like to thank the nursing staff of the hospital's ICU for their assistance.

\section{CONFLICT OF INTEREST}

None to declare.

\section{REFERENCES}

1. Potter BK, Burns TC, Lacap AP, Granville RR, Gajewski DA. Heterotopic ossification following traumatic and combatrelated amputations. Prevalence, risk factors, and preliminary results of excision. J Bone Joint Surg Am. 2007;89:476-86.

2. Sanchez-Duffhues G, Hiepen C, Knaus P, Ten Dijke P. Bone morphogenetic protein signalling in bone homeostasis. Bone 2015;80:43-59.

3. Grafe I, Alexander S, Peterson JR, et al. TGF-beta Family Signaling in Mesenchymal Differentiation. Cold Spring Harb Perspect Biol. 2018;10: a022202.

4. Kang $Q$, Song $W X$, Luo $Q$, et al. A comprehensive analysis of the dual roles of BMPs in regulating adipogenic and osteogenic differentiation of mesenchymal progenitor cells. Stem Cells Dev. 2009;18:545-59.

5. Luu HH, Song WX, Luo $X$, et al. Distinct roles of bone morphogenetic proteins in osteogenic differentiation of mesenchymal stem cells. J Orthop Res. 2007;25:665-77.

6. Wang YK, Sun WF, Liu XG, et al. Comparative study of serum levels of BMP-2 and heterotopic ossification in traumatic brain injury and fractures patients. Zhongguo Gu Shang. 2011;24:399-403.

7. Kang $H$, Dang $A B$, Joshi SK, et al. Novel mouse model of spinal cord injury-induced heterotopic ossification. J Rehabil Res Dev. 2014;51:1109-18.

8. Hofbauer LC, Schoppet M. Clinical implications of the
osteoprotegerin/RANKL/RANK system for bone and vascular diseases. JAMA 2004;292:490-5.

9. Boyce BF, Xing L. Functions of RANKL/RANK/OPG in bone modelling and remodelling. Arch Biochem Biophys. 2008;473:139-46.

10. Boyce BF, Xing L, Shakespeare W, et al. Regulation of bone remodelling and emerging breakthrough drugs for osteoporosis and osteolytic bone metastases. Kidney Int Suppl. 2003:S2-5.

11. Sherman AL, Williams J, Patrick L, Banovac K. The value of serum creatine kinase in early diagnosis of heterotopic ossification. J Spinal Cord Med. 2003;26:227-30.

12. Singer M, Deutschman CS, Seymour CW, et al. The Third International Consensus Definitions for Sepsis and Septic Shock (Sepsis-3). JAMA 2016;315:801-10.

13. Argyropoulou MI, Kostandi E, Kosta P, et al. Heterotopic ossification of the knee joint in intensive care unit patients: early diagnosis with magnetic resonance imaging. Crit Care, 2006;10:R152.

14. Yano M, Kawao N, Okumoto K, Tamura Y, Okada K, Kaji H. Fibrodysplasia ossificans progressiva-related activated activinlike kinase signalling enhances osteoclast formation during heterotopic ossification in muscle tissues. J Biol Chem. 2014;289:16966-77.

15. Lounev VY, Ramachandran R, Wosczyna MN, Yamamoto $M$, Maidment AD, Shore EM, et al. Identification of progenitor cells that contribute to heterotopic skeletogenesis. J Bone Joint Surg Am. 2009;91:652-63.

16. Scott MA, Levi B, Askarinam A, et al. Brief review of models of ectopic bone formation. Stem Cells Dev. 2012;21:655-67.

17. Tanaka H, Nagai E, Murata $H$, et al. Involvement of bone morphogenic protein-2 (BMP-2) in the pathological ossification process of the spinal ligament. Rheumatology 2001;40:1163-8.

18. Suzuki T, Bessho K, Segami N, Nojima T, lizuka T. Bone morphogenetic protein-2 in temporomandibular joints with internal derangement. Oral Surg Oral Med Oral Pathol Oral Radiol Endod. 1999;88:670-3.

19. Zhou N, Li Q, Lin X, et al. BMP2 induces chondrogenic differentiation, osteogenic differentiation and endochondral ossification in stem cells. Cell Tissue Res. 2016;366:101-11.

20. Qu X, Chen Z, Fan D, Xiang S, Sun C, Zeng Y, et al. Two novel BMP-2 variants identified in patients with thoracic ossification of the ligamentum flavum. Eur J Hum Genet 2017;25:565-571.

21. Yu PB, Deng DY, Lai CS, et al. BMP type I receptor inhibition reduces heterotopic [corrected] ossification. Nat Med. 2008;14:1363-9.

22. Micha D, Voermans E, Eekhoff MEW, et al. Inhibition of TGFbeta signalling decreases osteogenic differentiation of fibrodysplasia ossificans progressiva fibroblasts in a novel in vitro model of the disease. Bone 2016;84:169-180.

23. Agarwal S, Loder SJ, Breuler C, et al. Strategic Targeting of Multiple BMP Receptors Prevents Trauma-Induced Heterotopic Ossification. Mol Ther. 2017;25:1974-1987.

24. Wu J, Ren B, Shi F, Hua P, Lin H. BMP and mTOR signalling 
Available online at: www.jccm.ro

in heterotopic ossification: Does their crosstalk provide therapeutic opportunities? J Cell Biochem. 2019;120:1210812122.

25. Al-Fakhri N, Hofbauer LC, Preissner KT, Franke FE, Schoppet $M$. Expression of bone-regulating factors osteoprotegerin (OPG) and receptor activator of NF-kappaB ligand (RANKL) in heterotopic vascular ossification. Thromb Haemost. 2005;94:1335-7.

26. Bucay N, Sarosi I, Dunstan CR, et al. Osteoprotegerin-deficient mice develop early onset osteoporosis and arterial calcification. Genes Dev. 1998;12(9):1260-8.
The Journal of Critical Care Medicine 2021;7(1) • 45

27. Wilkinson JM, Stockley I, Hamer AJ, Barrington NA, Eastell R. Biochemical markers of bone turnover and development of heterotopic ossification after total hip arthroplasty. J Orthop Res. 2003;21:529-34.

28. Hudson SJ, Brett SJ. Heterotopic ossification--a long-term consequence of prolonged immobility. Crit Care, 2006;10:174.

29. Eastell R, Szulc P. Use of bone turnover markers in postmenopausal osteoporosis. Lancet Diabetes Endocrinol. 2017;5:908-923.

30. Fatayerji D, Eastell R. Age-related changes in bone turnover in men. J Bone Miner Res. 1999;14:1203-10. 